\title{
Combined inhibition of rho-associated protein kinase and EGFR suppresses the invasive phenotype in EGFR-dependent lung cancer cells
}

\author{
Ijeoma Adaku Umelo ${ }^{\mathrm{a}}$, Olivier De Wever ${ }^{\mathrm{b}}$, Peter Kronenberger ${ }^{\mathrm{a}, \mathrm{c}}$, Alfiah Noor $^{\mathrm{a}}$, \\ Erik Teugels ${ }^{\mathrm{a}}$, Gang Chen ${ }^{\mathrm{a}}$, Marc Bracke ${ }^{\mathrm{b}}$, Jacques De Grève ${ }^{\mathrm{a}, *}$ \\ a Laboratory of Molecular Oncology and Department of Medical Oncology, Oncologisch Centrum, Universitair Ziekenhuis Brussel, Belgium \\ ${ }^{\mathrm{b}}$ Laboratory of Experimental Cancer Research and Department of Radiation Therapy and Experimental Cancer Research, Universitair Ziekenhuis Ghent, \\ Belgium \\ ${ }^{\mathrm{c}}$ Laboratory for Biotechnology, Department of Healthcare, Erasmushogeschool Brussel, Brussels, Belgium
}

\section{A R T I C L E I N F O}

\section{Article history:}

Received 3 June 2015

Received in revised form 11 August 2015

Accepted 15 August 2015

\section{Keywords:}

Non-small cell lung cancer

Invasion

ROCK

EGFR

Y-27632

Afatinib

\begin{abstract}
A B S T R A C T
Introduction: Lung cancer remains the leading cause of cancer-related mortality worldwide, with metastatic disease frequently a prominent feature at the time of diagnosis. The role of NSCLC-derived EGFR mutations in cancer cell proliferation and survival has been widely reported, but little is known about the function of these mutations in invasive growth and metastasis. In this study, we sought to evaluate the intrinsic invasive properties of NSCLC cells with differing EGFR status and examine possible therapeutic targets that can abrogate invasive growth.

Materials and methods: Collagen-based assays and 3D cell cultures were used to assess morphological features, actin cytoskeleton dynamics and the invasive capacity of NSCLC cell lines with differing EGFR status. The role of the RhoA/ROCK/MYPT1 and EGFR/HER pathways in NSCLC-related invasion was investigated by pharmacological inhibition and RNA interference techniques.

Results: We demonstrate a positive correlation between EGFR mutational/amplification status and invasive capacity. Knockdown of wild-type and mutant EGFR leads to depletion of active and total MYPT1 levels. Combined pharmacological inhibition or genetic ablation of ROCK/EGFR suppresses the hallmarks of cancer cells and abrogates the invasive phenotype in EGFR-dependent NSCLC cells.

Conclusions: These observations suggest that combined targeting of the ROCK and EGFR/HER pathways may be a potential therapeutic approach in limiting invasive growth in NSCLC.
\end{abstract}

(c) 2015 Elsevier Ireland Ltd. All rights reserved.

\section{Introduction}

Lung cancer is the leading cause of cancer-related mortality worldwide, with local invasion and distal metastases as a prominent feature at time of presentation [1]. The invasive process is the result of a complex interaction between cancer cells and the host tissue environment, actin cytoskeleton dynamics, degradation of matrix barriers and the formation of membrane protrusions and

\footnotetext{
* Corresponding author at: Laboratory of Medical and Molecular Oncology, Department of Medical Oncology, Oncology Center, UZ Brussel, Vrije Universiteit Brussel (VUB), Laarbeeklaan 101, 1090 Brussels, Belgium. Fax: +32 24776210.

E-mail addresses: iumelo@vub.ac.be (I.A. Umelo), Olivier.DeWever@UGent.be (O.D.Wever), Peter.kronenberger@vub.ac.be (P. Kronenberger), alfinoor@vub.ac.be (A. Noor), Erik.Teugels@uzbrussel.be (E. Teugels),gangchen@vub.ac.be (G. Chen), Marc1.Bracke@UGent.be (M. Bracke), Jacques.Degreve@uzbrussel.be (J.D. Grève).
}

other migratory features necessary for invasive growth [2]. In the same perspective, advances in cancer biology have also shown that genomic alterations of the human epidermal growth factor receptor (EGFR/HER) play a critical role in cancer progression and tumorigenesis in a broad spectrum of human cancers including non-small cell lung cancer (NSCLC) [3]. The role of these NSCLC-derived EGFR mutations in cancer cell proliferation, and survival has been widely studied [4-6]; however studies demonstrating their specific role in the context of the invasive phenotype have so far been limited.

The Rho-associated coiled-coil forming protein (ROCK) is a known regulator of actin cytoskeletal organization, involved in Rho-induced formation of actin stress fibers and focal adhesions [7]. These actomyosin structures play an important role in cellular contractility and as a consequence also in cell adhesion, migration and morphogenesis. There is emerging evidence indicating that the closely related ROCK1 and ROCK2 kinases support the invasive 
a

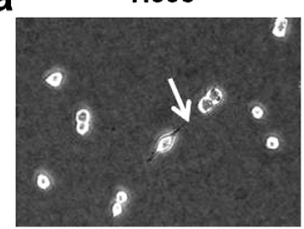

b

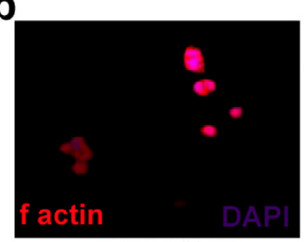

EGFR wild-type
H1650
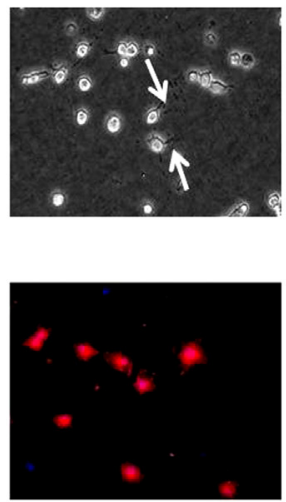

EGFR del(E746- A750)
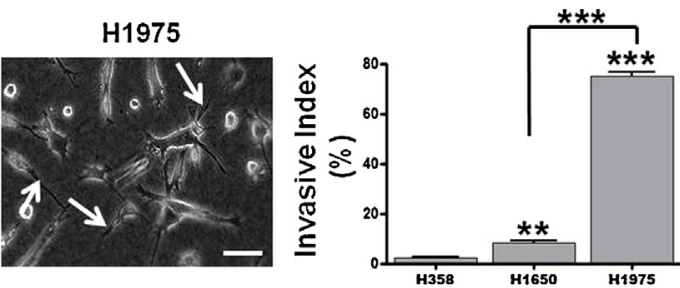

C
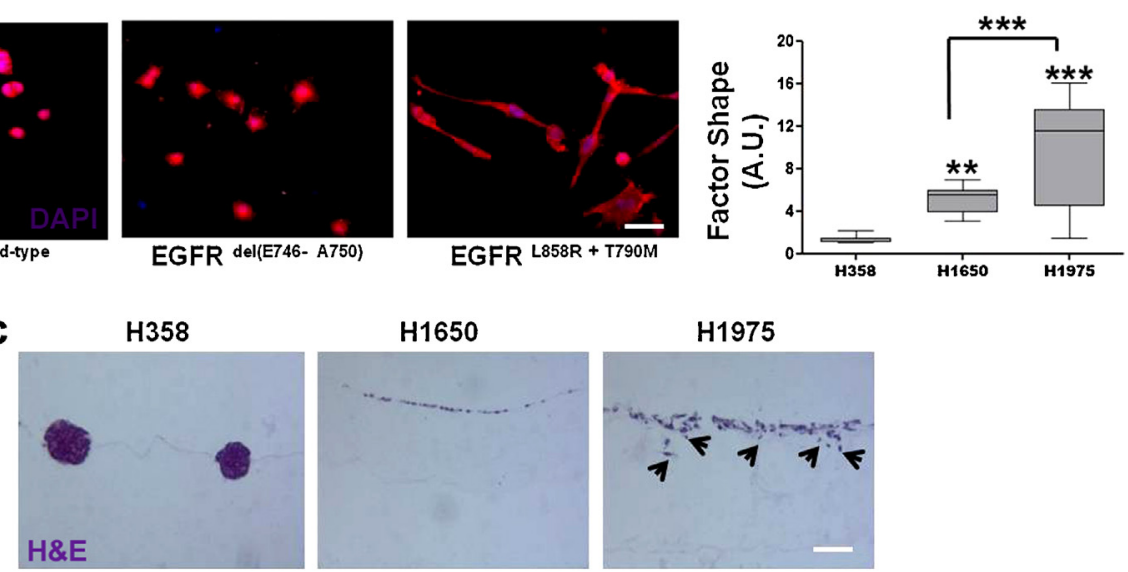

H1975
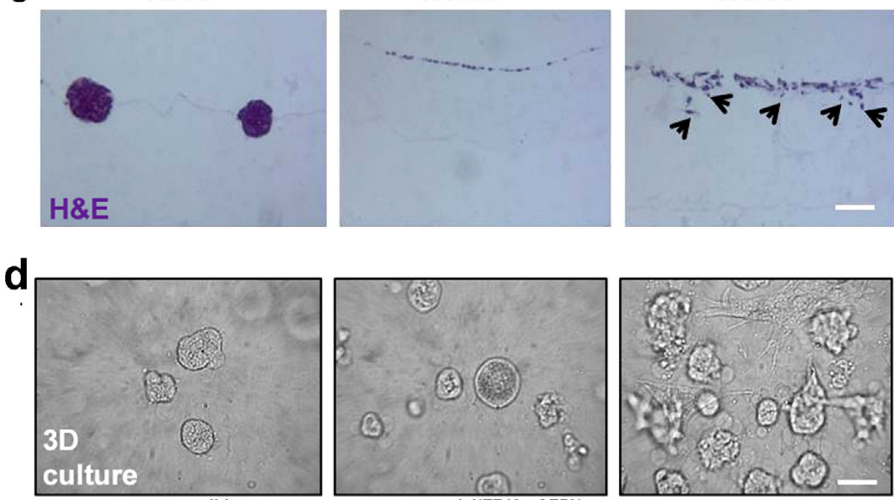

EGFR wild-type
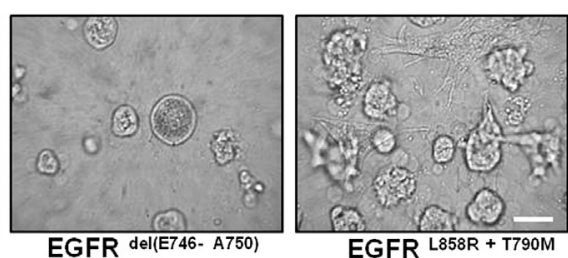

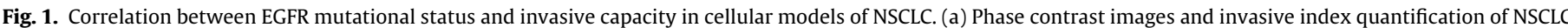

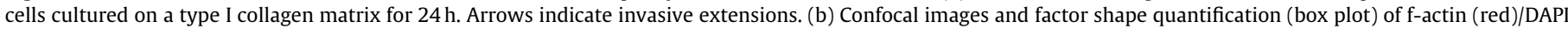

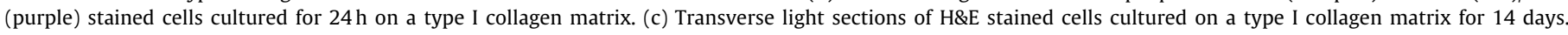

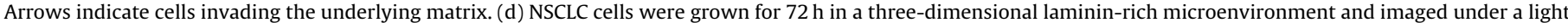
microscope. Scale bars, $50 \mu \mathrm{m}$.

and metastatic phenotype in a variety of cancers including lung cancer [8]. However, the biological role of the ROCK proteins in NSCLC invasion and metastasis is unclear. Here, we investigated the intrinsic invasive capacity of NSCLC cell lines with differing EGFR mutational/amplification status and examine the effect of combined EGFR/ROCK inhibition on the invasion and survival of NSCLC cells with differential invasive capacities.

\section{Results}

\subsection{Correlation between EGFR mutational status and invasive capacity}

To determine a possible correlation between EGFR mutational status and invasion, we first compared the intrinsic invasive capacity of three NSCLC cell lines harboring differential EGFR status. After a $24 \mathrm{~h}$ culture on a native type I collagen substrate, H1975 NSCLC cells carrying both a primary EGFR ${ }^{\text {L858R }}$ activating mutation and a secondary EGFR ${ }^{\mathrm{T} 790 \mathrm{M}}$ tyrosine kinase inhibitor (TKI) resistance-conferring mutation [8], displayed a more spread cellular morphology with the formation of cellular extensions inside the matrix (invasive index: $75 \pm 1.7 \%$ ). In contrast, H1650 (EGFR $\triangle$ E746-A750) and H358 (EGFR ${ }^{\mathrm{wt}}$ ) NSCLC cells attached as rounded cells with a significantly lower invasive index (invasive index: $8.3 \pm 0.7 \%$ and $2.3 \pm 0.3 \%$ respectively; Fig. 1 a). In addition, PC-9 NSCLC cells bearing the same exon 19 deletion mutation as $\mathrm{H} 1650$ cells (EGFR $\left.{ }^{\triangle E 746-A 750}\right)$, displayed a comparable invasive index of
$12.2 \pm 0.9 \%$; while H1781 cells harboring wild-type EGFR and a HER2 insertion mutation did not form invasive extensions on a collagen matrix (Supplemental Fig. 1a). Interestingly, A549 NSCLC cells harboring a wild-type EGFR amplification were categorized as highly invasive, with an invasive index of $72 \pm 2.5 \%$ (Supplemental Fig. 1b).

To examine differences in cortical cytoskeleton and cell surface morphology, the panel of NSCLC cell lines were stained for f-actin (by the toxin phalloidin) after a $24 \mathrm{~h}$ culture on type I collagen. As shown in Fig. 1b, H1975 cells displayed a more polarized morphotype with multiple long protrusions whereas H1650 and H358 cells assumed a rounded morphology with cortical F-actin and the appearance of membrane blebs. As shown in Supplemental Fig. 1c, A549 cells displayed a similar morphological phenotype as H1975 cells, with a disrupted and polarized cortical f-actin cytoskeleton.

We further evaluated the intrinsic invasive capacity of H1975 NSCLC cells, by examining their invasive depth in a type I collagen matrix. NSCLC cells were cultured on a collagen for 14 days, whereby transverse sections were analyzed for the presence of cells invading the collagen substrate. Our results show H1975 cells infiltrating into the collagen matrix whereas $\mathrm{H} 1650$ cells are confined to a single cellular layer and H358 cells form colonies at the top of the collagen (Fig. 1c).

To establish a cell culture that more closely reflects an in vivo lung cancer microenvironment, we characterized the NSCLC cell lines in laminin-rich three-dimensional cultures. As shown in Fig. 1d, H1975 cells appeared as colonies showing extensive con- 
a

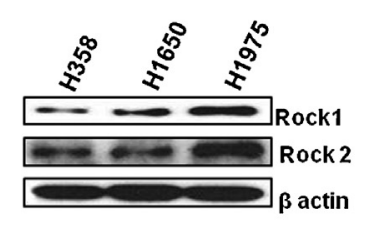

b

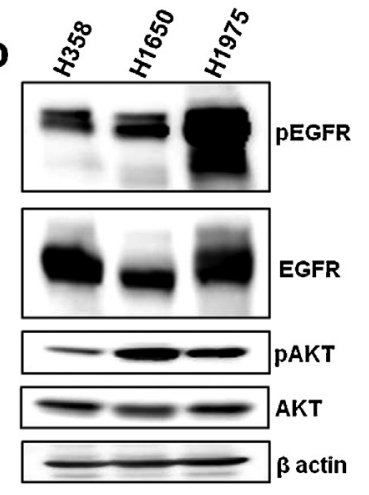

C

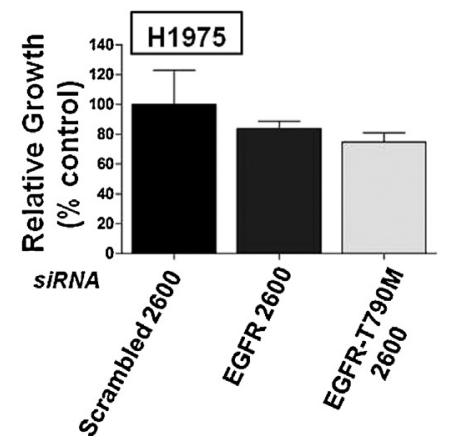

d

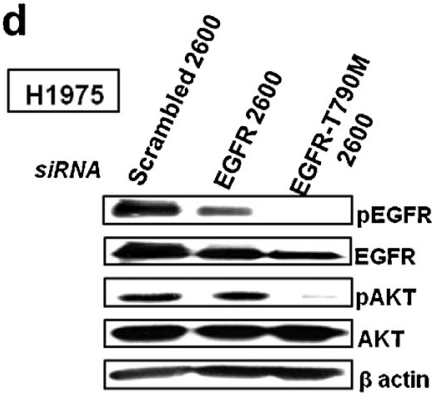

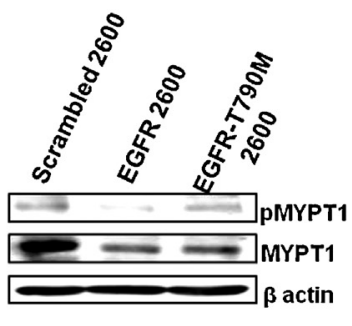

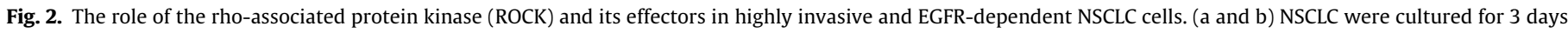

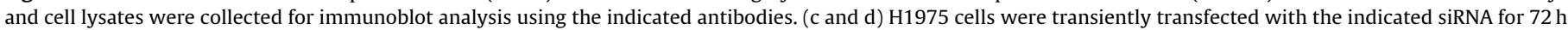
and assessed for growth by MTS analysis. Total cell lysates were collected for immunoblot analysis using the indicated antibodies.

necting extensions, suggestive of direct adhesion between the cells and the extracellular matrix (ECM). In contrast, H358 and H1650 cells appeared as colonies that were situated apart in the ECM (Fig. 1d).

\subsection{Role of rho-associated coiled coil forming protein (ROCK) and its effectors in highly invasive EGFR mutant NSCLC cells}

Overexpression of the ROCK proteins has been implicated in the invasive and metastatic potential of various tumor types [9-11]. Baseline protein expression of ROCK1 and ROCK2 was higher in H1975 cells compared to H1650 and H358 NSCLC cells (Fig. 2a), indicating that the ROCK protein may be a crucial factor in the invasive phenotype of $\mathrm{H} 1975$ cells. EGFR activation status was also greatly enhanced in H1975 cells compared to the other cell lines; possibly due to the presence of the EGFR ${ }^{\mathrm{T} 790 \mathrm{M}}$ mutation in this cell line (Fig. 2b). The EGFR ${ }^{\mathrm{T} 790 \mathrm{M}}$ mutation is associated with acquired resistance to EGFR TKI therapy and has been found to increase EGFR activity as well as oncogenicity in NSCLC $[12,13]$.

Accumulating lines of evidence have demonstrated that AKT activation correlates with enhanced tumor invasion [14,15]. AKT phosphorylation was also significantly higher in H1975 cells compared to H358 cells, but H1650 cells exhibited even higher AKT phosphorylation compared to the other two tested cell lines (Fig. 2b). This observed high AKT signaling in H1650 cells may be the result of the combined effect of the EGFR mutation and a PTEN null status, which has been implicated in deregulated AKT activity [16].

To elucidate the role of the EGFR ${ }^{\mathrm{T} 790 \mathrm{M}}$ mutation in the invasive properties of $\mathrm{H} 1975$ cells, we performed RNA interference experiments. EGFR ${ }^{\mathrm{T} 790 \mathrm{M} 2600}$-specific mediated-knockdown effected a $\sim 25 \%$ reduction in cell growth $72 \mathrm{~h}$ post-transfection in concordance with our previous findings [17], while EGFRWT2600 siRNA reduced cell growth by $\sim 15 \%$ at the same time point (Fig. 2 c). By $72 \mathrm{~h}$ post-transfection, we observed a more distinct reduction in both phosphorylated and total EGFR levels as well as active AKT after EGFR ${ }^{\text {T790M2600 }}$ siRNA-mediated knockdown compared to both EGFR $^{\text {WT2600 }}$ and scrambled ${ }^{\text {T790M2600 }}$ treatment (Fig. 2d). Further analysis on the effect of wild-type and mutant EGFR -mediated knockdown on ROCK expression did not reveal a reduction in their protein levels (data not shown). Interestingly, however, wild-type and mutant EGFR siRNA treatment clearly reduced the levels of myosin phosphastase target subunit 1 (MYPT1); a direct target of ROCK (Fig. 2d). These data thus suggest that EGFR inhibition could play a role in suppressing the invasive phenotype of H1975 cells.

\subsection{Effect of ROCK and EGFR inhibition on the growth of NSCLCS with differing EGFR mutational status}

As both ROCK1 and ROCK2 are highly expressed in H1975 cells (see Fig. 2a), we sought to determine the effects on growth of Y27632, an investigational inhibitor of the ROCK family of kinases that competes with ATP for binding to its catalytic cleft [18]. In addition, we also investigated the inhibitory effects of afatinib, an EGFR/HER family inhibitor that covalently and irreversibly binds to the ATP binding cleft of the tyrosine kinase and also has some activity in T790 M cell lines [19]. Dose-dependent analysis of the growth inhibitory effect of Y-27632 in the panel of NSCLC cells (H358, H1650, H1975 and A549) demonstrates that Y-27632 was unable to achieve an $\mathrm{IC}_{50}$ value even at a concentration of $10 \mu \mathrm{M}$ (Supplemental Fig. 2a). On the other hand, we observed a striking to modest growth inhibitory effect with afatinib in the majority of the 

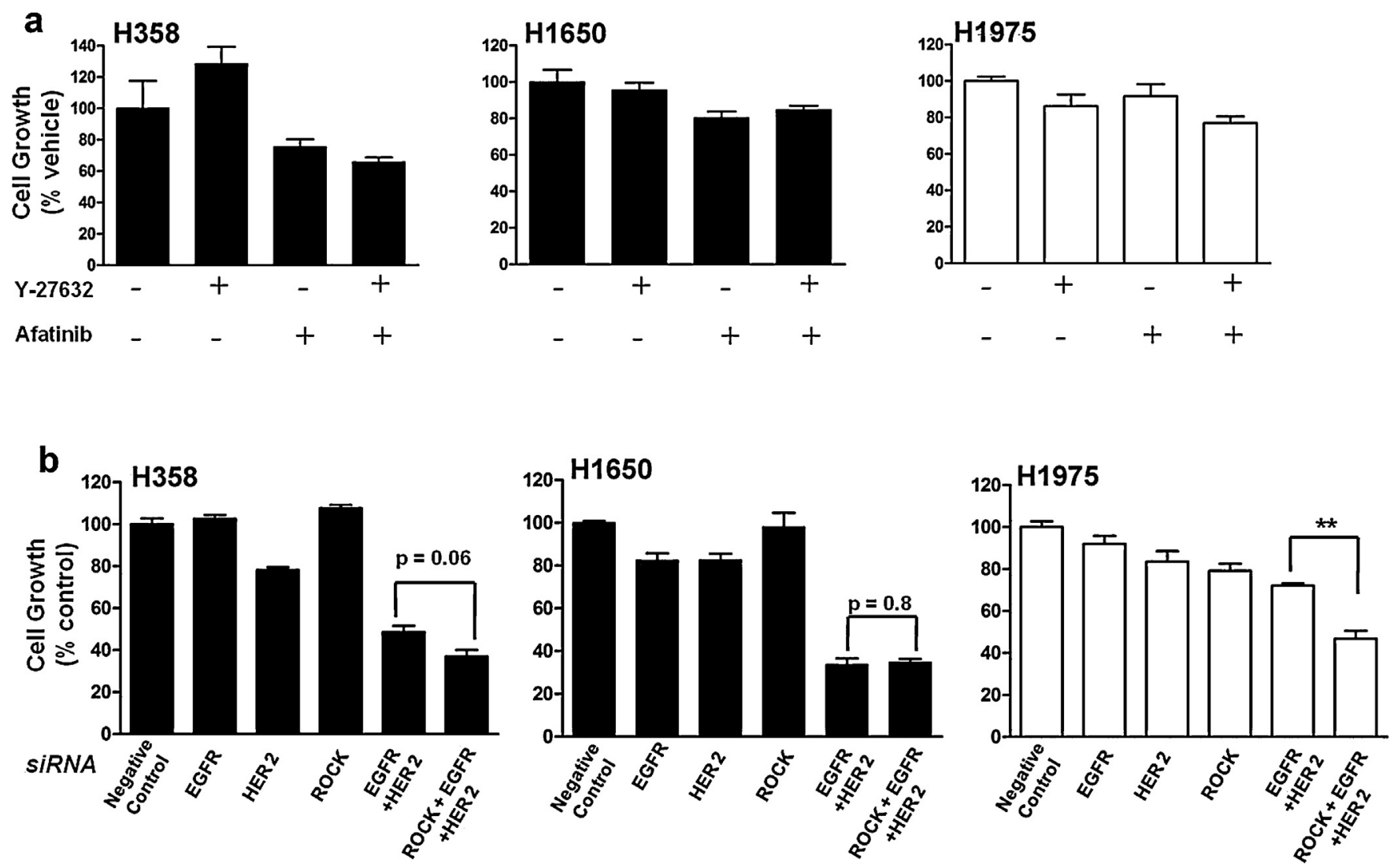

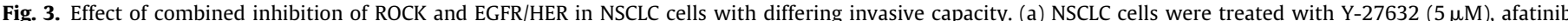

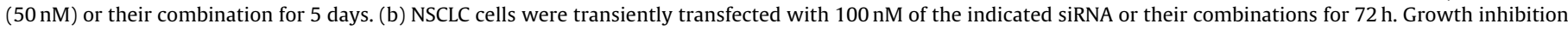
was assessed by MTS analysis.

cell lines tested (Supplemental Fig. 2b). Treatment with afatinib did not significantly reduce the growth of A549 cells at clinically relevant doses (Supplemental Fig. 1b).

To investigate the combined effect of EGFR and ROCK kinase inhibition we treated the respective NSCLC cell lines (H358, H1650 and H1975) for 5 days with Y-27632 and afatinib as either single agents or combined. Our results display modest effects of the Y-27632/afatinib combination on the growth of both H358 and H1975 cells when compared to single inhibitor treatments (Fig. 3a), while we observed no additional effect when Y-27632 was added to afatinib in H1650 cells (Fig. 3a). While combined treatment with Y-27632/afatinib did not reduce the growth of A549 cells compared to vehicle control, fasudil (a clinically active ROCK inhibitor) combined with afatinib achieved modest effects in this cell line (Supplemental Fig. 1d). Interestingly, fasudil monotherapy also potently reduced the ability of A549 cells to form colonies in soft agar compared to combined treatment (Supplemental Fig. 1e).

We subsequently performed siRNA transfection experiments to further verify our pharmacological findings. We show that the addition of ROCK siRNA treatment potently reduces cell growth in the H1975 and H358 cells $72 \mathrm{~h}$ posttransfection when compared to combined EGFR/HER2 or single EGFR or HER2 siRNA treatments (Fig. 3b). Consistent with the pharmacological experiments, no significant additional effect was obtained when ROCK-specific siRNA was added to the EGFR/HER2 siRNA combination in H1650 cells (Fig. 3b)

\subsection{Effect of ROCK and EGFR inhibition on the invasive phenotype of EGFR mutant NSCLC cells}

To further assess the efficacy of the Y-27632/afatinib combination, we evaluated their effect in abrogating the invasive phenotype in H1975 cells. Our results show that Y-27632 combined with afatinib was superior to either agent alone in the reduction of both invasive index and factor shape after a $24 \mathrm{~h}$ treatment on a type I collagen substrate (Fig. 4a and b). Moreover, this combination significantly reduced the amount of H1975 cells infiltrating into the collagen after a 14 day inhibitor treatment (Fig. 4c). Importantly, we also observed a marked decrease in the number of H1975 cells invading the collagen substrate with single Y-27632 treatment compared to the effects seen with single treatment with afatinib (Fig. 4c). The effect of Y-27632 on H358 non-invasive cells was less striking after a similar 14 day treatment on a collagen substrate. Afatinib treatment coincided with robust suppression in the amount of colonies formed by H358 cells, while treatment with Y-27632 did not yield significant effects (Supplemental Fig. 3a).

Combined treatment of Y-27632 and afatinib reduced phosphorylated levels of ROCK protein activators and effectors (RhoA and MYPT1) and potently suppressed AKT phosphorylation in H1975 cells (Fig. 4d and Supplemental Fig. 3b). In addition, while afatinib treatment reduced active levels of EGFR and HER2 in H1975 cells, active ERK levels were cooperatively reduced with combination treatment (Supplemental Fig. 3c). Both Y-27632 and afatinib had an effect on cell survival, after a 5 day treatment, but the effect was not enhanced when Y-27632 was added to afatinib (Fig. 4e). To support our pharmacological data, we examined the effect EGFR/HER2/ROCK siRNA treatment on actin cytoskeleton morphol- 

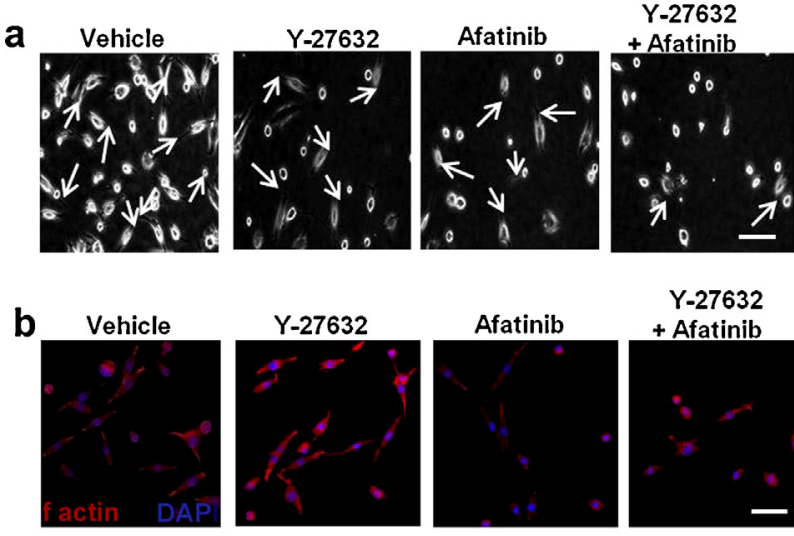

Y-27632 + Afatinib

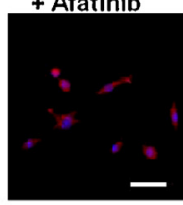

C

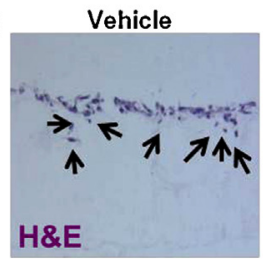

Afatinib

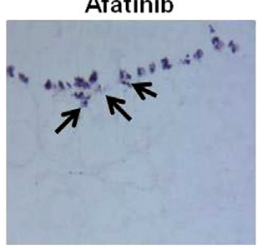

$\mathrm{Y}-27632$

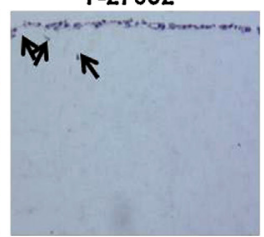

$\mathrm{Y}-27632$

+ Afatinib

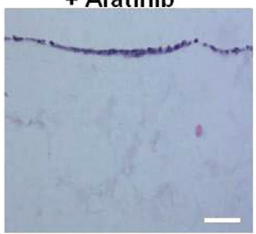

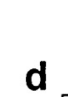

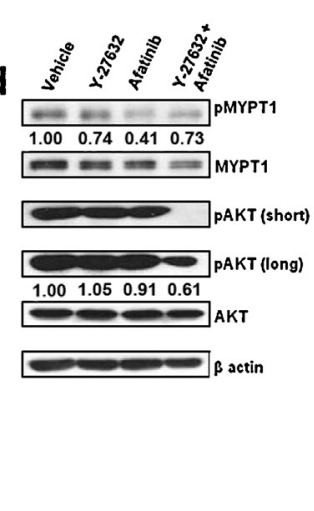

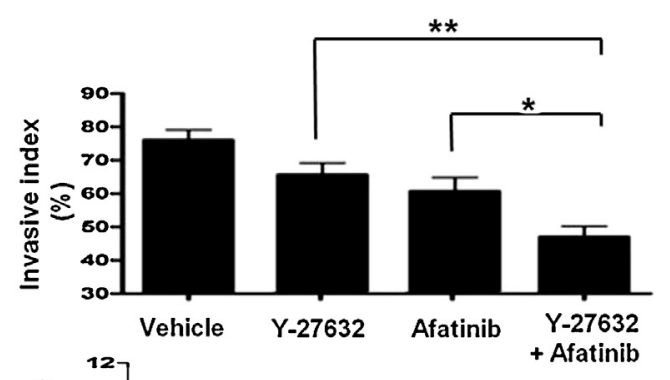
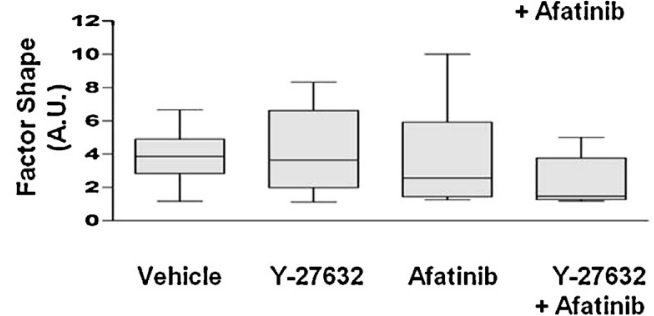

e
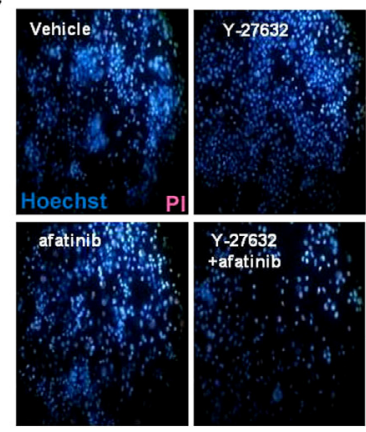

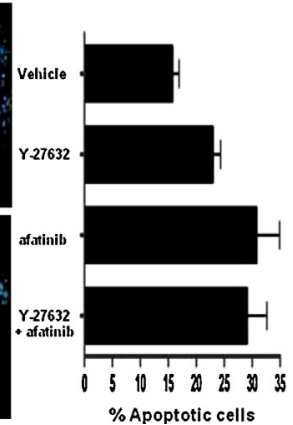

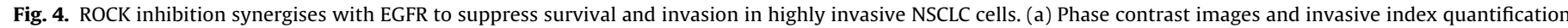

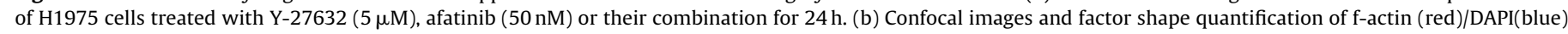

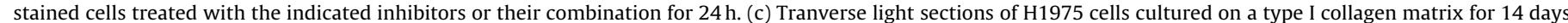

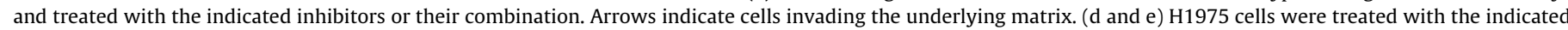

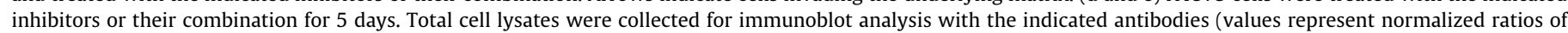

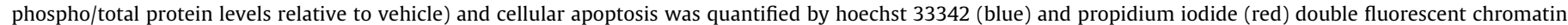
staining. Bars, $50 \mu \mathrm{m}$.(For interpretation of the references to color in this figure legend, the reader is referred to the web version of this article.).

ogy of H1975 cells. H1975 cells remained with a polarized F-actin morphology after siRNA-mediated depletion of EGFR (Supplemental Fig. 3d). However, depletion of HER2 promoted a more rounded morphology with combined depletion of EGFR, HER2 and ROCK further promoting an organized F-actin cytoskeleton (Supplemental Fig. 3d).

\section{Discussion}

In this study we evaluated the intrinsic invasive properties of NSCLC cells with differing EGFR status, and examined the biological role of the Rho/ROCK pathway in regulating the function of highly invasive cells. Our data demonstrates that both EGFR and ROCK play a role in the growth and invasion of NSCLC cells with different EGFR genotypes. The combined effects of ROCK and EGFR inhibition on the growth and invasive capacity of highly invasive H1975 cells (EGFR ${ }^{\mathrm{L} 858 \mathrm{R}+\mathrm{T} 790 \mathrm{M}}$ ) suggests that dual targeting of the ROCK and EGFR/HER proteins may offer a potential therapeutic benefit.

Our findings reveal that NSCLC cells carrying a wild-type EGFR status do not promote invasion and point to a positive correlation between an invasive phenotype and a constitutively active mutant EGFR or a wild-type EGFR amplification status. A recent study by Talasila et al., support these observations, where amplification or activation of wild-type EGFR were considered to be important factors in the invasive phenotype of mouse models of glioblastoma [20].
Our findings also indicate that in highly invasive NSCLCs, the ROCK pathway is activated and that mutant EGFR plays a role in this (Fig. 5). The activation level of the highly expressed ROCK protein in these cells causes a striking response to both EGFR/HER2 and RhoA/ROCK/MYPT1 inhibition resulting in the suppression of both survival and invasion (Fig. 5). In addition, RhoA/ROCK signaling plays a pivotal role in actin cytoskeleton dynamics and tumor cell motility [21] and our findings also highlight that combined EGFR/ROCK inhibition corresponds with changes in cell morphology and the reorganization of filamentous actin in highly invasive and EGFR-dependent NSCLC cells. AKT activation correlates with enhanced tumor invasion [14,15], and based on the presented results dual ROCK/EGFR treatment antagonizes AKT signaling; which similarly leads to the robust suppression of MAPK/ERK pathway activity. It must be noted, however, that while the ROCK1 and ROCK2 proteins share significant sequence homology, they have been described to exert different functions in the cell [22,23]. ROCK1 is reported to be involved in actin cytoskeleton destabilization while ROCK2 has been shown to be an essential factor in stabilizing the actin cytoskeleton. The observed suppressive effects on invasion may thus result from inhibition of the ROCK1 protein, as pharmacological inhibitors of ROCK equally inhibit the activity of both ROCK1 and ROCK2 [24].

Several preclinical studies have demonstrated that inhibition of Rho/ROCK in cells bearing oncogenic forms of KIT, FLT3 and BCR-ABL TK reduces active levels of MYPT1 and suppresses their constitutive growth [25]. A publication by Burthem and colleagues 


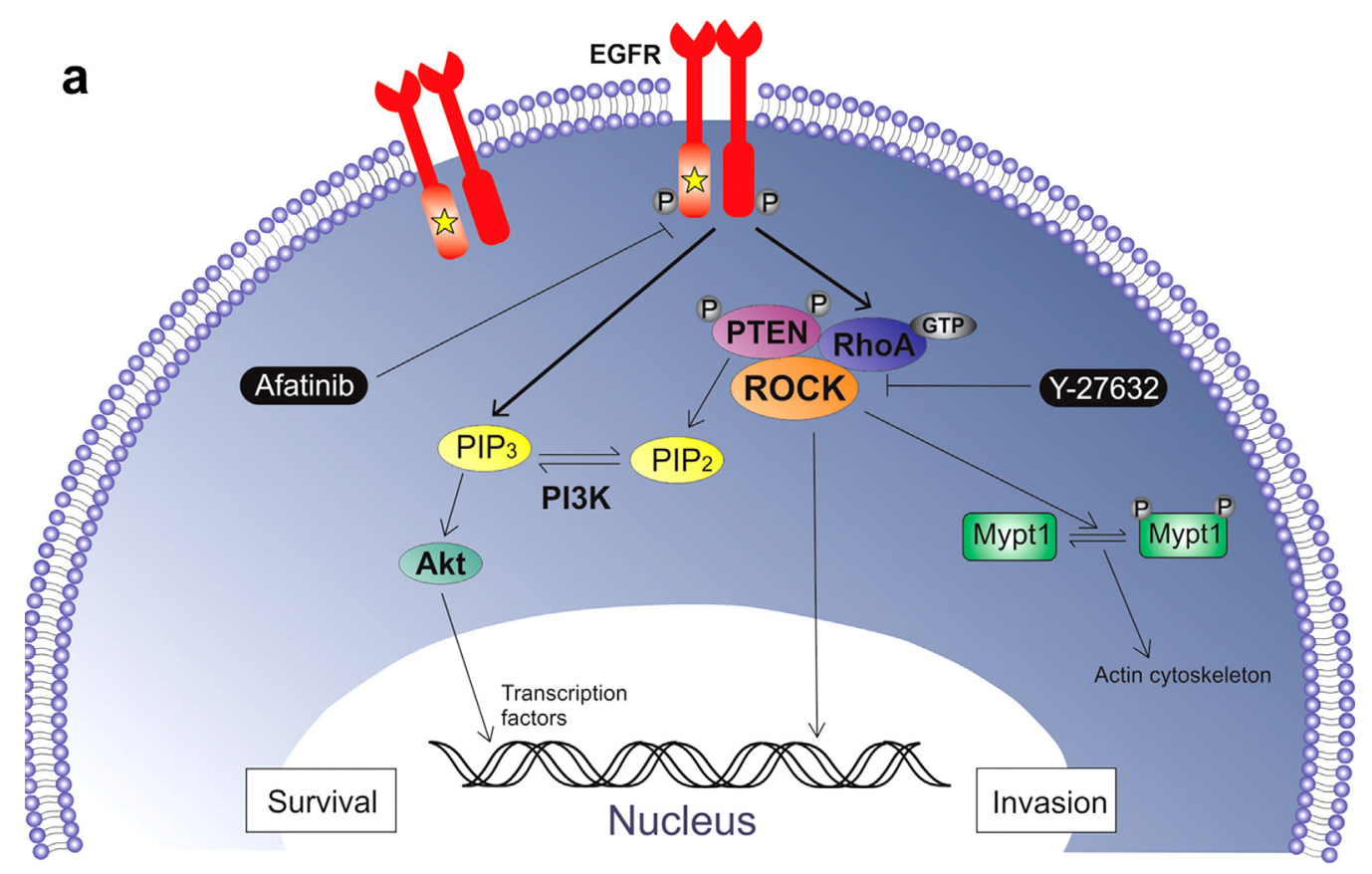

b

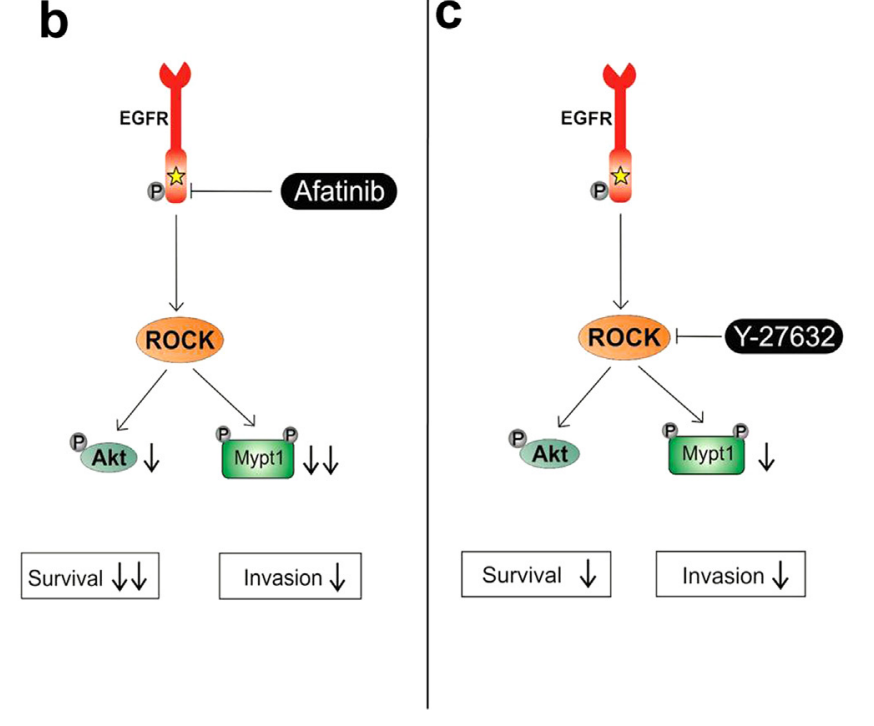

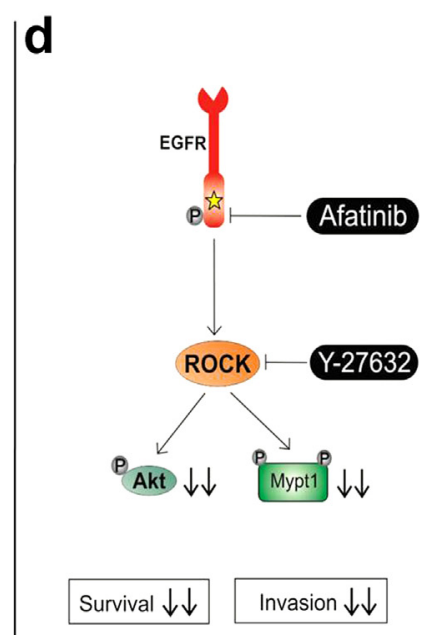

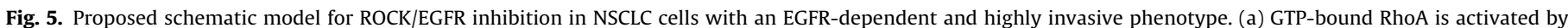

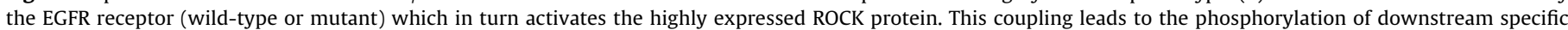

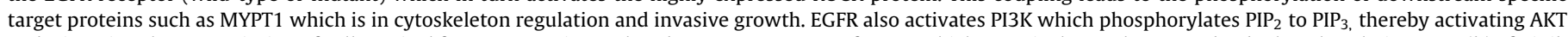

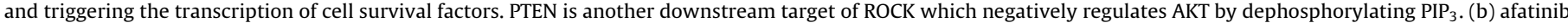

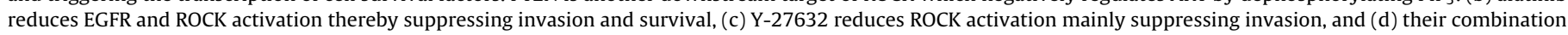
cooperatively suppresses both survival and invasion.

shows that ROCK inhibitors such as Y-27632 and fasudil combined with the TKI imatinib synergistically inhibits the activity of human leukemic cells [26]. These results show that ROCK may be a potential additional therapeutic target in impairing the oncogenic activity of TK-driven malignancies and validate the use of these inhibitors as either single agents or combined with other TKIs as a possible therapeutic strategy. Since TKIs such as afatinib and imatinib are currently indicated for the treatment of some cancers and ROCK inhibitors such as fasudil have been effectively used for several years in the treatment of various diseases [27], it may be promising to test these combinations in future clinical trials [28].

In conclusion, our findings demonstrate that the ROCK family of protein kinases is important for highly invasive NSCLC can- cers. Whereas the functional role of the genetically altered EGFR receptor in the context of NSCLC pathogenicity has been widely described [29,30], our findings further demonstrate that mutational activation of EGFR may play a role in the invasive phenotype. The combined targeting the Rho/ROCK and EGFR/HER pathways should be further explored.

\section{Materials and methods}

\subsection{Cell lines and reagents}

Y-27632 (Selleckchem) and afatinib (Boehringer Ingelheim) were of highest grade available. The human NSCLC cell lines H358, 
H1650, H1975 were obtained from the ATCC and cultured as described [6]. The A4549 human NSCLC cell line was obtained from Sigma and cultured according to the suppliers recommendations. All cell lines were authenticated by STR-analysis.

\subsection{Invasion assays}

The Collagen invasion assay was performed as previously described [7]. Collagen matrices were imaged under a phasecontrast light microscope and the invasive index was subsequently determined by manual counting of the number of invading cells and non-invading cells present in 10-15 randomly selected fields.

Cell morphology was studied by determination of factor shape of minimal 25 single cells. Collagen matrices were fixed in 3\% paraformaldehyde and stained with Alexa Fluor 594 conjugated phallodin with a DAPI nuclei counterstain [31]. Imaging was subsequently performed by confocal microscopy.

For invasive depth, collagen matrices were fixed with buffered formalin, embedded in paraffin and $6-\mu \mathrm{m}$ sections were taken every $250 \mu \mathrm{m}$ over a total distance of $1500 \mu \mathrm{m}$. All sections were stained with hematoxylin and eosin (H\&E) and subsequently imaged under a light microscope. One representative field from one section out of 6 is shown.

\subsection{Three-dimensional cell cultures}

The NSCLC cells $\left(1 \times 10^{4}\right)$ were seeded in a Matrigel (Corning) suspension in clear-bottomed 96 well plates. The Matrigel suspension was kept at $37^{\circ} \mathrm{C}$ until polymerization and a top layer of growth medium was then added. Cells were maintained in Matrigel for 3 days and imaging was performed with a standard light-microscope to examine morphological differences.

\subsection{Western blot analysis and antibodies}

Cells were lysed in a Tris-buffer containing a protease and phosphatase inhibitor cocktail (Sigma). Lysates were cleared by centrifugation and protein concentration was determined by the Bradford protein assay kit (Bio-Rad). Equivalent amount of protein were loaded on a resolving acrylamide gel and blotted on a polyvinylidene fluoride membrane (PVDF). The membrane was then subjected to an immunodetection procedure using the indicated antibodies. HRP-conjugated secondary antibodies (GE Healthcare) and a chemiluminescent detection kit (Advansta) were used to detect the indicated proteins.

\subsection{Growth assays}

Cell growth was assessed by the colorimetric tetrazolium MTS assay (Promega). Cells were seeded in clear-bottomed 96-well plates at a density of $2 \times 10^{3}$ cells/well in triplicates. For drug inhibition studies, cells were treated after $24 \mathrm{~h}$ with various concentrations of the indicated drugs or their combinations. After the indicated time periods, the numbers of viable cells were analyzed using an ELISA reader (Labsystems).

\subsection{RNA interference}

Cells were seeded into 24 -well or 96 -well plates at a density of approximately $1 \times 10^{5}$ cells/well or $5 \times 10^{3}$ cells/well. $24 \mathrm{~h}$ later, cells were transfected with siRNA against EGFR WT2600, EGFR $^{\mathrm{T} 790 \mathrm{M} 2600}$ and scrambled EGFR T790M2600 [17]; EGFR, HER2, Genome Non-Targeting siRNA (Thermo Scientific); and ROCK (Qiagen) using Lipofectamine 2000 (Invitrogen) according to the manufacturer's instructions.

\subsection{Hoechst 3342/propidium Iodide staining}

The effects of the inhibitors on apoptosis and nuclear morphology of H1975 cells was assessed by Hoechst 33342 and propidium iodide (PI) double fluorescent chromatin staining as described [32].

\subsection{Statistical analysis}

Results are representative of three independent experiments. All graphical data is presented as the mean \pm standard error of mean (SEM). The Chi-Square test was utilized for group comparison of invasive index while the Mann Whitney test was utilized when comparing factor shape. Statistical significance is reported as follows: ${ }^{*}, P<0.05,{ }^{* *}, P<0.01$ and ${ }^{* * *}, P<0.001$.

\section{Conflict of interest statement}

The authors declare no conflict of interest.

\section{Acknowledgments}

This work was supported by the National Cancer Plan (Belgium), the Stichting Tegen Kanker (Belgium) and a Vrije Universiteit Brussel PhD fellowship.

\section{Appendix A. Supplementary data}

Supplementary data associated with this article can be found, in the online version, at http://dx.doi.org/10.1016/j.lungcan.2015.08. 008.

\section{References}

[1] C.S. Dela Cruz, L.T. Tanoue, R.A. Matthay, Lung cancer: epidemiology, etiology, and prevention, Clin. Chest Med. 32 (4) (2011) 605-644.

[2] M. Yilmaz, G. Christofori, EMT, the cytoskeleton, and cancer cell invasion, Cancer Metastasis Rev. 28 (1-2) (2009) 15-33.

[3] S.V. Sharma, J. Settleman, ErbBs in lung cancer, Exp. Cell Res. 315 (4) (2009) $557-571$

[4] J. Jiang, et al., Epidermal growth factor-independent transformation of Ba/F3 cells with cancer-derived epidermal growth factor receptor mutants induces gefitinib-sensitive cell cycle progression, Cancer Res. 65 (19) (2005) 8968-8974.

[5] R. Sordella, et al., Gefitinib-sensitizing EGFR mutations in lung cancer activate anti-apoptotic pathways, Science 305 (5687) (2004) 1163-1167.

[6] R. Mulloy, et al., Epidermal growth factor receptor mutants from human lung cancers exhibit enhanced catalytic activity and increased sensitivity to gefitinib, Cancer Res. 67 (5) (2007) 2325-2330.

[7] A.L. Bishop, A. Hall, Rho GTPases and their effector proteins, Biochem. J. 2 (Pt. (348)) (2000) 241-255

[8] K. Riento, A.J. Ridley, Rocks: multifunctional kinases in cell behaviour, Nat. Rev. Mol. Cell Biol. 4 (6) (2003) 446-456.

[9] T. Kamai, et al., Significant association of Rho/ROCK pathway with invasion and metastasis of bladder cancer, Clin. Cancer Res. 9 (7) (2003) 2632-2641.

[10] J. Lane, et al., The expression and prognostic value of ROCK I and ROCK II and their role in human breast cancer, Int. J. Oncol. 33 (3) (2008) 585-593.

[11] D. Rosel, et al., Up-regulation of Rho/ROCK signaling in sarcoma cells drives invasion and increased generation of protrusive forces, Mol. Cancer Res. 6 (9) (2008) 1410-1420.

[12] C.H. Yun, et al., The T790M mutation in EGFR kinase causes drug resistance by increasing the affinity for ATP, Proc. Natl. Acad. Sci. U. S. A. 105 (6) (2008) 2070-2075.

[13] W. Pao, et al., Acquired resistance of lung adenocarcinomas to gefitinib or erlotinib is associated with a second mutation in the EGFR kinase domain, PLoS Med. 2 (3) (2005) e73.

[14] V. Vasko, et al., Akt activation and localisation correlate with tumour invasion and oncogene expression in thyroid cancer, J. Med. Genet. 41 (3) (2004) 161-170.

[15] A. Dudley, et al., DRR regulates AKT activation to drive brain cancer invasion, Oncogene 33 (41) (2014) 4952-4960.

[16] M.L. Sos, et al., PTEN loss contributes to erlotinib resistance in EGFR-mutant lung cancer by activation of Akt and EGFR, Cancer Res. 69 (8) (2009) 3256-3261.

[17] G. Chen, et al., Effect of siRNAs targeting the EGFR T790M mutation in a non-small cell lung cancer cell line resistant to EGFR tyrosine kinase 
inhibitors and combination with various agents, Biochem. Biophys. Res. Commun. 431 (3) (2013) 623-629.

[18] T. Ishizaki, et al., Pharmacological properties of Y-27632: a specific inhibitor of rho-associated kinases, Mol. Pharmacol. 57 (5) (2000) 976-983.

[19] F. Solca, et al., Target binding properties and cellular activity of afatinib (BIBW 2992), an irreversible ErbB family blocker, J. Pharmacol. Exp. Ther. 343 (2) (2012) 342-350.

[20] K.M. Talasila, et al., EGFR wild-type amplification and activation promote invasion and development of glioblastoma independent of angiogenesis, Acta Neuropathol. 125 (5) (2013) 683-698.

[21] N. Rath, M.F. Olson, Rho-associated kinases in tumorigenesis: re-considering ROCK inhibition for cancer therapy, EMBO Rep. 13 (10) (2012) 900-908.

[22] J. Shi, et al., Distinct roles for ROCK1 and ROCK2 in the regulation of cell detachment, Cell Death Dis. 4 (2013) e483.

[23] F.E. Lock, et al., Differential regulation of adhesion complex turnover by ROCK1 and ROCK2, PLoS One 7 (2) (2012) e31423.

[24] S. Vemula, et al., ROCK1 functions as a suppressor of inflammatory cell migration by regulating PTEN phosphorylation and stability, Blood 115 (9) (2010) 1785-1796.
[25] R.S. Mali, et al., Rho kinase regulates the survival and transformation of cells bearing oncogenic forms of KIT: FLT3, and BCR-ABL, Cancer Cell 20 (3) (2011) 357-369.

[26] J. Burthem, et al., The rho-kinase inhibitors Y-27632 and fasudil act synergistically with imatinib to inhibit the expansion of ex vivo CD34(+) CML progenitor cells, Leukemia 21 (8) (2007) 1708-1714.

[27] M.F. Olson, Applications for ROCK kinase inhibiton, Curr. Opin. Cell Biol. 20 (2) (2008) 242-248

[28] N. Rath, M.F. Olson, Rho-associated kinases in tumorigenesis: re-considering ROCK inhibition for cancer therapy, EMBO Rep. 13 (10) (2012) 900-908.

[29] T. Mitsudomi, Y. Yatabe, Epidermal growth factor receptor in relation to tumor development: EGFR gene and cancer, FEBS J. 277 (2) (2010) 301-308.

[30] S.V. Sharma, et al., Epidermal growth factor receptor mutations in lung cancer, Nat. Rev. Cancer 7 (3) (2007) 169-181.

[31] O. De Wever, et al., Modeling and quantification of cancer cell invasion through collagen type I matrices, Int. J. Dev. Biol. 54 (5) (2010) 887-896.

[32] G. Chen, et al., Targeting the epidermal growth factor receptor in non-small cell lung cancer cells: the effect of combining RNA interference with tyrosine kinase inhibitors or cetuximab, BMC Med. 10 (2012) 28. 